\title{
Towards a Reference Architecture for Female-Sensitive Drug Management
}

\author{
Deborah Mateja \\ University of Mannheim \\ mateja@uni-mannheim.de
}

Lukas-Valentin Herm

Julius-Maximilians-Universität Würzburg

lukas-valentin.herm@uni-wuerzburg.de

\author{
Evita Bartels \\ Tilburg University \\ E.A.Bartels@tilburguniversity.edu
}

\author{
Luis Oberste \\ University of Mannheim \\ oberste@uni-mannheim.de
}

\author{
Alexa Danelski \\ Freie Universität Berlin \\ a.danelski@fu-berlin.de
}

\begin{abstract}
Due to various biological factors, males and females differ in their response to drug treatment. However, there is still a lack of knowledge of the effects resulting from sex-differences in the medical field, primarily due to females' underrepresentation in clinical studies. Considering severe diseases related to the cardiovascular system, which are likely to be perilous, counteracting this lack and emphasizing the need for sex-dependent drug treatment is of high importance. Thus, this research-in-progress paper aims to strengthen the female perspective in drug management by proposing design considerations on IS regarding recommender systems in healthcare to reinforce shared decision-making and person-centered care. The resulting artifact presented will be a reference architecture with a mobile application as the interface to patients and healthcare professionals as well as a data-driven backend to collect and process data on sex specificity in the medical treatment of cardiovascular diseases (CVD).
\end{abstract}

\section{Introduction}

Despite the increasing awareness that sex-related differences between males and females need to be considered in drug treatment (e.g., [1], [2]), there is a persisting lack of knowledge on varying drug tolerance based on the sex of the patient, especially for females (e.g., [3][4]). Biological differences between males and females lead to differences in the pharmacokinetics and the pharmacodynamics, in the drug absorption, in the drug distribution in tissues, in the metabolism or the differing excretion via the kidney and intestine [3]. The biological causes for these differences lie predominantly in the unequal genetic constitution of males and females, diverse body conditions - e.g., females tend to have a lower body mass index, a smaller size of organs and more adipose tissue - and in the impact of sexual hormones [5][3]. Additionally, several studies showed that the adverse side effects of medical drug use are more frequent for females than males [1]. Thus, there is an obvious need for sex-dependent drug treatment. However, females are still underrepresented in clinical trials. Even in the field of animal testing drugs are predominantly tested on male animals assuming that sex differences play no role in the clinical effect [3]. In this regard, Karlsson-Lind et al. (2017) show that information on sex differences lacks for a variety of available medications [1]. Also, Regitz-Zagrosek (2004) depicts that males and females differ in their willingness to participate in their treatment process actively and women tend to take different amounts of possibly interacting over-the-counter drugs for selfmedication in addition to prescribed drugs [3].

Especially for severe diseases such as CVDs, which are the most relevant cause of mortality and morbidity worldwide, sex-related cardiovascular risk-factors and respective drug administration are essential determinants of treatment success [5]. However, there is a lack of knowledge for female-specific needs in medical treatment, which accordingly can become extremely dangerous [6].

All those factors emphasize the importance of gaining fundamental knowledge on female-specific needs in drug management, especially in CVD. Thus, our study proposes design consideration for information systems (IS) focusing on strengthening the female perspective in drug administration, regarding aspects such as female-specific side-effects and drug dosage and potential drug interactions. Specifically, the focus is on the decision-making process for the medical treatment of CVD patients. With this, we aim to contribute design knowledge on how to identify, communicate, and handle female-specific CVD drug responses. While female patients are our primary stakeholders, healthcare professionals need to be considered and are the primary 
decision-makers in medical treatment. Also, as the sex inequity in medical treatment arises from narrow research, both from academia and the drug industry, they are essential stakeholders to close the persisting knowledge gap on sex-specificities in medical treatment. Our study focuses on Germany as a target country.

The paper is structured as follows: First, we introduce core socio-technical and technical foundations. Second, we review the literature to carve out the knowledge gap and gain insights into the solution space. Third, we introduce our general design science research methodology. Fourth, we describe the first iteration of our proposed IS artifact and the first evaluation cycle through a quantitative survey. Last, we discuss our results and limitations as well as introduce the further course of our study.

\section{Theoretical background}

\subsection{Personalized healthcare}

Tailoring services towards the individual is an essential element of healthcare delivery [7]. Various concepts address how to do this, such as personalized medicine, person-centered care, and shared decisionmaking. Personalized medicine "encompasses the application of genomic and molecular data to target better the delivery of healthcare and more holistic lifelong approaches." [8, p. 208]. However, Di Paolo et al. (2017) discovered that most personalized medicine literature focuses on tailoring treatments to patients' diseases without considering patients' needs, beliefs, behavior, values, wishes, utilities, environment, and circumstances [7]. Patient preferences were hardly considered when evaluating new medicines rather than focusing on the stratification according to individual biological information. As a response to that, personcentered care focuses on the person as a whole and is responsive towards the person's individual needs and preferences [9]. To achieve person-centered care, mutual involvement, and collaboration of the healthcare professional with the patient are required [10]. This collaboration process is referred to as shared decisionmaking. Shared decision-making is "an approach where clinicians and patients share the best available evidence when faced with the task of making decisions, and where patients are supported to consider options, to achieve informed preferences" [10, p. 1361]).

\subsection{Explainable recommender systems in healthcare}

Recommender systems are tools to provide users with suggestions for action [11]. With the digitalization in almost all areas of daily life and the resulting increase in data volume and complexity, these systems assist users in dealing with this through condensing relevant information to generated recommendations. This is also evident in the healthcare industry [12]. To develop recommender systems, three different techniques are typically used [11].

(1) Collaborative filtering is the process of clustering people with similar usage profiles. Likewise, this approach can be applied across similar application areas [13]. (2) Knowledge-based filtering uses existing knowledge about the user, such as demographics [11]. (3) Content-based filtering compares different products that the user has already used to estimate their similarity and propose new products accordingly [14]. Nevertheless, the implementation of only one of these solutions can lead to the so-called "cold start problem". Due to an information gap, the recommender system cannot develop a precise recommendation [15]. Therefore, the use of hybrid recommender systems is a frequently used approach. Within these systems, at least two decision strategies are combined to identify recommendations [16].

To process a large amount of data through the recommender system, various artificial intelligence (AI) methods are used in healthcare today [13]. These approaches mostly suggest a recommendation similar to collaborative filtering but in a more precise manner [17]. AI refers, besides others, to the concept of machine learning (ML) and deep learning (DL). These models are trained in a data-driven way using mathematical procedures without explicit programming [18].

Simultaneously, several problems are associated with these AI-methods. One main problem is the complexity of the generated models resulting in blackbox models [19]. This black box model is not traceable and, therefore, leads to trust issues. Consequently, this lack of trust reduces the acceptance and willingness to use such a recommender system [20]. The explainable AI (XAI) research field attempts to address this issue while preserving AI's benefits [21]. As trust is a core element in healthcare, explainability is a frequently targeted goal for AI systems in healthcare research [22].

\section{Methodology}

Our study aims to support women diagnosed with CVD in their medical treatment decision-making process. Specifically, this study reports on a first 
iteration of the problem-centered approach to the Design Science Research Methodology proposed by Peffers et al. (2007) [23]. Further iterations will follow in subsequent studies.

Identify problems \& define objectives. We started the first design cycle by investigating sex differences in medical treatment and the under-representation of females in clinical trials. Hereby, we regarded the needs of female patients as well as the demands of healthcare professionals within the treatment decision-making process (e.g., [1], [24]). For this, relevant concepts were identified to shape the solution space: For sociotechnical challenges faced through sex differences in medical treatment, the concepts of shared decisionmaking (e.g., [10]) and person-centered care (e.g., [25], [26]) were identified. For the technical perspective on supporting shared-decision-making and personcentered care, explainable hybrid recommender systems (e.g., [13], [27]) were chosen as a foundation.

Relevant literature is consulted and condensed to a precise research gap and core findings to be considered in the solution space based on a structured literature review following the approach of Webster and Watson (2002) [28]. We aim to find extant contributions dealing with recommender systems in healthcare for shared decision-making. We analyze the databases PubMed, ScienceDirect, and Web of Science to find healthcarerelated contributions. Regarding technical contributions, we examine ACM Digital Library and IEEE Xplore. For the field of business information systems, AIS eLibrary is consulted. The following search term pseudocode is used:

((health care | care) AND (shared decision-making | patient-cent* | person-cent*) AND (gender equity | gender disparity | gender differences | gender bias) AND (recommend* system | decision support system) AND (explainab* recommend* $\mid$ artificial intelligence | machine learning $\mid$ deep learning $\mid X A I)$ )

Thereby, we were able to identify 5454 contributions. Through a meta-analysis, full-text analysis as well as a forward and backward search, we classify 46 contributions as relevant. The results are presented in Chapter 4.

Design and development. To tackle sex differences in medical treatment, this study proposes a reference architecture as an artifact to support females in their medical treatment decisions. Specifically, the identified core concepts are used as a basis for a set of design decisions regarding the required functionality of such an artifact. These socio-technical design decisions are then transferred into a set of technical design decisions prescribing a possible implementation for the targeted artifact. This reference architecture serves as a foundation for implementing such an artifact in the specific context of females with CVD sickness. The results are presented in Chapter 5.

Evaluation. In this first iteration of the design cycle, we evaluate the relevance of the problem space and the basic idea of the reference architecture to generate datadriven female-specific recommendations on medical treatment. The evaluation is conducted as a quantitative survey with 43 German females at 35 years of age or older, presented in Chapter 6.

Communication. In this first design cycle, we propose a reference architecture as an artifact rooted in extant research. The artifact can be considered an exaptation contribution [29] as it tackles a new problem with established concepts and solutions. With this first iteration of the design cycle, we posit a research-inprogress paper that will eventually yield a nascent design theory contributing design knowledge to general healthcare IS and sex-sensitive IS. Future research will build on these initial steps.

\section{Literature review}

The literature review aims to find extant contributions dealing with recommender systems in healthcare for shared decision-making to understand the targeted gap better and identify reusable concepts.

Person-centered care and sex differences. Personcentered care benefits both the patient, e.g., through enhanced knowledge, improved relationship with providers, service experience and satisfaction, treatment compliance and health outcomes, as well as the health system, e.g., through cost-effective service delivery [30]. Nevertheless, many patients, especially females, do not experience person-centered care due to a lack of insight into operationalizing person-centered care for females [31]. One explanation is the lack of attention paid to person-centered care in medical guidelines [30]. Multiple strategies are proposed to improve personcentered care for females, including patient-oriented tools in guidelines. These tools can, for example, include information about the condition, lifestyle advice, psychological strategies, and strategies for communicating with healthcare professionals.

An observational study by Meijers (2019) showed that the application of shared decision-making in practice increased between 2007 and 2015 [32]. Despite this increase, the level of shared decision-making is still limited and leaves room for improvement. Another study using the same measurement instrument in cardiac stress testing found no significant sex effect [33].

A system allowing patients to access their medical records maintained by a healthcare facility supports patients to have an active role in their care process [34]. Ideally, this system also allows patients to interact with 
their healthcare providers. Currently, the management of chronic conditions often relies on face-to-face visits and infrequent measurements [35]. This may cause a delay when interventions are needed since patients tend to wait until symptoms are acute. Late detection of serious symptoms can lead to expensive healthcare services, requiring hospitalization, emergency room visits, and unplanned readmissions. Early detection of deteriorating health is therefore needed to improve health outcomes and manage healthcare costs. Mobile health has shown to impact health-related behaviors, allowing patient self-management of their health status. An example of a mobile health app monitoring and analyzing health data of persons with and without chronic diseases, and automatically alerting care support staff based on purpose-developed algorithms is the one developed in the study by Petersen (2019). The app has three functional areas: health data, personal profile, and secure messaging. The health data was recorded through a fitness tracker smartwatch and data entered by the patients manually. The personal profile involved information on the patient's personal preferences, health goals, and social data. This information is useful for healthcare providers to deliver person-centered care. Secure messaging was possible $24 / 7$ with a healthcare professional. The pilot demonstrated that persons with chronic conditions were actively engaged for a longer time than persons without chronic diseases. Besides, the pilot uncovered substantial potentials for the reduction in care costs for persons with chronic care conditions.

Recommender systems. The necessity of recommender systems in healthcare is frequently discussed in research. Contributions such as [36] highlight the need to integrate shared decision-making with interactive recommender systems as the most appropriate way to raise patient awareness. Therefore, their application can be found in various approaches such as telehealth [37], chatbots [36], or mobile applications [41]. These approaches are also used to address different areas of healthcare, such as diets [38], drug treatment [39], dementia [40], support for the elderly [41], or chronic diseases [42]. The described recommender systems mostly use a hybrid collaborative filtering technique to overcome the cold start problem [42]. According to these contributions, mobile applications differentiate themselves from others by their high flexibility and acceptance, enabling the elicitation of sensor and fitness data as an additional source of information [43].

Furthermore, the review also shows that until 2017, different cluster algorithms were mainly used for the prediction of recommender systems. Due to the high computational complexity, these contributions also introduce outsourcing to cloud services [44]. On the other hand, recent contributions focus on more complex algorithms such as DL or natural language processing due to their high predictive power and are also used in high-stakes areas like radiology. Therefore, those recommendations can support physicians in the drafting of reports [45]. Simultaneously, the examination of recent studies in this field reveals the necessity of traceability of the predictions. Especially in critical areas such as cancer detection [36]. Even in the preliminary contributions, this has already been discussed by pointing out decision factors of neural networks when recommending treatments [46].

Identified knowledge gap. The analysis proves the necessity of paying attention to how to improve sex equity and shared decision-making in medical practice. Several strategies and solutions were highlighted. An already established approach is the use of recommender systems through the application of mHealth, based on explainable AI. However, in the analysis, no contributions could be identified dealing with the development of IS for shared decision-making to tackle the prevailing sex differences despite the frequently discussed relevance of this gap [31]. Therefore, our study aims to develop a reference architecture to support further developments in this field.

\section{Designing female-sensitive personalized drug management}

In this section, we propose a set of means-end relations, i.e., appropriate means (M) to achieve ends (E) associated with the problem domain [47]. Those are derived from both technical and socio-technical causeeffect relations identified from the reviewed literature and inferred from the core concepts. These means-end relations guide not only the instantiation of the derived reference architecture, shown in Fig. 1, in our specific context but can also be reused for health-related IS in general.

\subsection{First design cycle}

Socio-technical considerations. Our approach to tackling the knowledge gap on sex differences in medical treatment evolves around increased patient participation. Therefore, the artifact must be designed to facilitate ease of use as a determinant for increased patient participation (e.g., [48]) (E1). Following our objective to develop an application that allows for female-centered care, as a specific manifestation of person-centered care, the literature emphasizes that it is vital to elicit and incorporate an individual's values and preferences (e.g., [25]). Therefore, the artifact has to provide a way to collect and store data about patient 


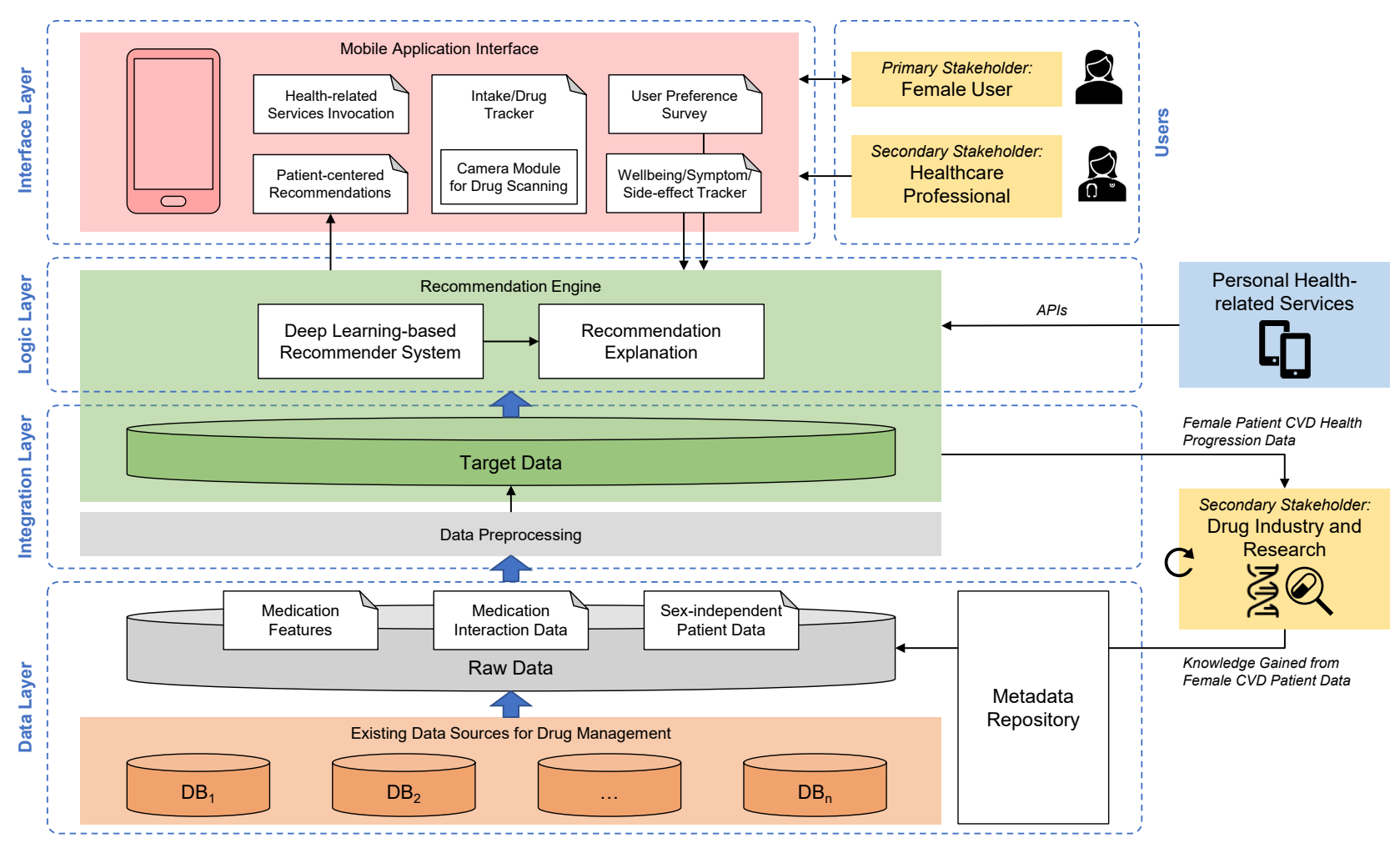

Figure 1. Reference architecture for female-sensitive drug management.

preferences (E2). With the aim of the artifact to enable a shared decision-making process, the literature points out the importance of sharing the best available evidence when facing the task of making a decision (e.g., [10]). Therefore, the artifact has to provide ways to track different types of information, including personcentered data, user preferences, and data on overall patient wellbeing, general healthcare-related data, and female-specific healthcare-related data (E3).

To deliver ground for a justified discussion to yield shared decision-making for medical treatment (e.g., [49]), the artifact has to provide a condensed account of the input data (E4). This information has to be shared with the patient to trigger shared decision-making and the healthcare professionals as a common ground for discussion [10] (E5). However, they require different degrees of information. While healthcare professionals need detailed explanation and reasoning, for example, about drug interactions, patients may rather be interested in whether and how their preferences can be mapped to their treatment (e.g., [49]). Hence, the artifact must display information with varying perspectives and degrees of detail per stakeholder (E6). Lastly, the general problem targeted by the artifact arises from the shortage of female-specific health-related data. Therefore, the artifact also shares the female-specific data collected by the artifact with academic and industry drug research (E7) to close the knowledge gap for sex differences in medical treatment.

Technical considerations. To increase patient participation, the artifact needs to be flexibly available to the patient anywhere, at any time [48]. Therefore, a mobile application was chosen as the appropriate interface for the patients (M1a). Moreover, ease of use is facilitated through functionalities, such as taking a picture of a medication instead of an interface requiring long and tiring inputs by the user [50] (M1b).

User preferences can be elicited by incorporating a patient survey. For the design of the survey, we follow extant work (e.g., [51]) (M2). To achieve M2, extant solutions incorporate various types of data. First, general healthcare-related data can contribute to the overall understanding of the interplay of drugs, symptoms, and side effects, either specific for CVD or general and either female-specific or sex-independent data as proposed by the literature stream on evidencebased medicine (e.g., [52]). This data can be made available to the artifact by establishing links to renowned healthcare-related databases, such as PharmacoGenomic Mutation Database (PGMD) [53] and the Medscape Interaction Checker [54]. Therefore, the artifact must provide linkages to healthcare-related databases (M3a). Second, extant solutions elicit personcentered data directly related to the sickness, such as monitoring drug intakes and data about the general well- 
being. Extant research frequently refers to this as 'patient profiling', including any information about activities and the progression of symptoms and sideeffects over time (e.g., [55]). Therefore, the artifact needs to provide users functionality to enter and track all data related to their patient profile (M3b). Moreover, Gyrard et al. (2020) propose to enhance patient-centered data with sensor data such as heart rates [13]. While this is not in the scope of our artifact, it may be available in other health-related applications. Other wellbeing and health-related applications, such as Apple Health or menstrual cycle calendars, may also collect insightful information and can deliver it to the artifact's recommendation engine if appropriately connected (e.g., [56], [52]). Therefore, the artifact should provide touchpoints to connect other health- and wellbeingrelated applications via APIs to retrieve further relevant data (M3c).

A highly frequented approach to condense information in healthcare is to provide recommendations based on the observed data within recommender systems (e.g., [39], [36]). Therefore, the artifact must provide a recommendation engine to condense all the observed data for the shared decisionmaking process (M4a). Initial recommender systems primarily rely on one type of data (e.g., transaction/behavioral data) and use the collaborative filtering method [57]. More advanced recommender systems additionally incorporate rather static data, such as preference profiles (e.g., [58], [59], [60]). For the artifact at hand, the recommender system ought to incorporate all the available data. Extant solutions exemplify how to incorporate user preferences [12], patient profiles (e.g., [55]), and external database (e.g., [52]). Therefore, the artifact should be designed as a hybrid recommender system to incorporate transactional data and user preferences (M4b). For recommender systems, their applications range from disease discovery [67] over suggestions for physical activity [60] to actual therapeutic recommendations [57], i.e., many types of recommendations are possible.

As a starting point, the artifact will provide recommendations on drug interactions, preferencebased alternations of drug intakes, and for scheduling an appointment with a healthcare professional in the case of irregularities (M4c). For any of these recommendations and any of the posited data sources, both female- and patient-specific as well as general health-related data, any recommender system faces the 'cold start problem' (e.g., [27], [16]). The hybrid approach allows us to propose initial recommendations based on a limited amount of data and then collect and learn from data accrued over time. Accordingly, initial recommendations may rely on sex-independent data and as the database grows over time, recommendations can become more tailored to female- and patient-specific needs. Also, with the database growing over time, more types of recommendations may be possible. A value can be created with a large and continuously growing database through so-called data network effects, where the multi-sided exchange among individual users allows to improve recommendations for each user [61]. Therefore, the artifact should stimulate the continuous collection and recording of data to evolve the recommendations continuously (M4d).

To achieve shared decision-making, the patient, who is in full possession of the mobile application and the data therein, can share information with the healthcare professional. Therefore, a login for healthcare professionals is provided in the artifact, where the patient can choose to share data explicitly with the healthcare professional (e.g., through using a QR code mechanism), so that the data remains secure (e.g., [37]) (M5). Recommendations require an explanation to be understood and followed by healthcare professionals and patients. Therefore, explainability is a core issue for any recommendation system in the healthcare context [22]. This issue is, for example, addressed by Che et al., 2015, who explain their DL-based mortality prediction [62]. Therefore, the artifact must provide sufficient explanations for each recommendation (M6a). Explanations must be adapted to stakeholders [63]. At the same time, a patient may be satisfied with a recommendation and indicators for how their preferences were considered and will be realized by a recommendation, a healthcare professional demands further details such as the specific interplay of components of drugs. Therefore, the artifact must provide different perspectives and granularities of explanations for each recommendation (M6b).

Lastly, the artifact wants to contribute the collected data such as female-specific symptoms, side-effects, sickness progressions, to the drug research, both academic and industry, to close the gap of sexdifferences in CVD treatment. Both storing and sharing health-related data raises data security issues, which must be addressed for the IS artefact to be viable. Therefore, the artifact provides an API to retrieve fully anonymized datasets from the backend (M7).

\subsection{First evaluation cycle}

In design science research, an evaluation is crucial to demonstrate the utility, quality, and efficacy of a proposed artifact [64]. While the designed reference architecture followed socio-technical requirements and was technically grounded on findings in extant research, we evaluate and plan to evaluate further both the relevance of the problem space as well as the suitability of the proposed solution. In this first evaluation cycle, 
we conducted a quantitative survey to verify the relevance of lacking sex-specific knowledge to females and whether females would like to be empowered through technology to better participate in their medical treatment decision-making process.

Method. A quantitative online survey was conducted from 19th to Jun. 25th, 20201. A pretest was conducted to check for clarity and correct spelling of the questions. The participants were reached via a recruiting pool, receiving incentives, as well as by using snowball sampling. Women of 35 years of age or higher were included in this German study. We applied a 5-point Likert scale ( $1=$ strongly disagree, $5=$ strongly agree). In total, 45 participants completed the survey, from which two low-effort responses were excluded from the analysis due to non-varying scale options; therefore, the sample group consists of 43 completed surveys.

Procedure. The survey opened with an introduction, addressing the lack of knowledge on sex differences in medical treatment. Along with eight questions, respondents were asked for their interest in receiving medical recommendations and their willingness to track their medical condition, share their data for recommendation processing, and forward personal health data to healthcare professionals and drug research. Also, their current use of health-related applications was inquired.

Results. Eighty-four percent of the respondents strongly agreed to wish to receive recommendations regarding drug interactions, preference-based alternations of drug intakes, and - in case of irregularities - to get in touch with a healthcare professional $(M=4.76, S D=0.55)$. This is in line with previous research on technology-enhanced, personcentered care reporting that patients with chronic conditions, such as CVD, are "more likely to remain engaged" with the technology [35, p. 10]. Receiving explanations in addition to the recommendation itself to understand better the effects of one's drug usage and influencing factors was strongly wished for as well $(M=4.67, S D=0.64)$. Thirty percent neither agreed nor disagreed with preferring a mobile application to collect and store personal health data, preferences, diseasespecific data, and data on one's overall well-being; $25.6 \%$ agreed $(M=3.00, S D=1.24)$. Regarding data sharing with healthcare professionals, $25.6 \%$ agreed and $44.2 \%$ strongly agreed $(M=3.93, S D=1.23)$. Sharing the data with academic and industrial research, was $37.2 \%$ agreed on and $30.2 \%$ strongly agreed on by participants $(M=3.16, S D=1.20)$. However, participants would rather not share their data with the recommender system for the sake of receiving more precise drug recommendations or - in equal shares - are regardless of that $(30.2 \%$ each, $M=2.65, S D=1.27)$. More than half of the participants already use a fitness app to track activities such as running or biking (55.8\%). A calendar app for the menstrual cycle is used by $23.3 \%$. The general perception of the respondents that sharing personal health-related data can help overcome the lack of sex equity in medical research was consensual $(M=$ 3.44, $S D=1.06)$.

Implications. The survey showed that respondents are willing to receive recommendations regarding their drug management. They also demand explanations for the provided recommendations and are willing to use the information for engaging in shared decision-making with their healthcare professionals. Also, they are willing to give away their data for research purposes to enhance female-specific medical treatment. However, in contrast to the high motivation to participate in shared decision-making for their medical treatment, they are reluctant to receiving improved recommendations to compensate for their data sharing. This implies that the adoption of such technology and, thus, the data collection, are more likely to succeed if users benefit from using the app through concomitant outcomes, i.e., tracking their health-record and getting detailed explanations on the observable data. Also, respondents do not explicitly consider a mobile application the preferred option to collect their health-related data. However, this is contrary to the result that more than half of the participants already use health-related mobile applications. In sum, the study shows that there is a need for female-sensitive, personalized recommendations and explanations for which data-driven solutions with female-specific design considerations are a promising path. Further research on socio-technical requirements is required to understand better female patient's needs as well as the utility of such applications in the daily routines of women to deduct specific sex-related requirements for the design of the proposed artefact.

\section{Discussion}

In this research-in-progress paper, we investigated design considerations for technology-enhanced femalesensitive decision-making regarding CVD's medical treatment. The result is a reference architecture with a mobile application as the interface to patients and healthcare professionals as well as a data-driven backend to collect and process data on sex differences in the medical treatment of CVD.

\footnotetext{
${ }^{1}$ See https://doi.org/10.13140/RG.2.2.27950.54086
} 


\subsection{Contributions to theory and practice}

From a theoretical perspective, there is a lack of knowledge on handling sex inequity in healthcare occurring through the underrepresentation of females in clinical trials. Our study aims to close this gap in three ways. First, we apply the concept of person-centered care with the aim of closely monitoring the body condition of each female patient to make data available on female-specific side effects, course of a disease, and general wellbeing under medication. Second, we apply the concept of shared decision-making to enable females to be more involved in their medical treatment decision despite the lack of research knowledge on femalespecificity. Third, we apply the technical concept of hybrid explainable recommender systems to realize female-sensitive drug management along with the concepts of person-centered care and shared decisionmaking to incorporate sex-differences in medical treatment.

From a practical view, the implementation of the reference architecture will serve female patients, healthcare professionals, and drug researchers. First, female patients are yet frequently not aware of sex inequity in clinical trials and what this implies for their medical treatment. Based on the mobile application, as the patient interface in the reference architecture, female patients can better understand how their preferences and body specificity can be considered in their medical treatment decision. Second, healthcare professionals benefit from the condensed data made available to them, primarily through the provided explanations, to allow them to make better-tailored treatment decisions for female patients. Third, the underrepresentation of females in clinical trials can be tackled through the rich records of female patient data accrued through the mobile application. With females accurately tracking their health-related data for their personal treatment decision, drug research can be pointed towards relevant areas of study to facilitate targeted investigation of female-specificity in drug research and, ultimately, to reduce the gap of female-specific knowledge for medical treatments.

\subsection{Future research}

For the further progression of this study, we plan to iterate further design cycles. The evaluation of our current research state yielded essential feedback for the next design phase. We currently develop and evaluate mock-ups as a second design cycle that allows us to get intermediate patient feedback between the conceptualization and implementation. Having finished the conceptualization, the data structure will be derived and defined based on relevant data, both from underlying drug management data sources and userrelated data, which are integrated into the recommendation engine. Subsequently, we plan to realize a first implementation of the artifact. Thereby, we can build up an essential user base and basic functionality such as data collection and recommendations based on existing medical interaction databases. Hereupon, the user base grows, and more female-specific health data becomes available, allowing to infer female-specific knowledge.

Based on these two upcoming design cycles, we seek to evaluate further aspects: The architecture feasibility will be verified through the actual implementation and functional software testing for each component, the user interface, and the recommendation engine. The sociotechnical requirements will be evaluated through explorative (female) focus groups to identify concrete sex-related requirements for the design of the proposed artefact to appropriately address the specific needs of the target group, the female patients, for a proper interaction with the application. The utility of recommendations will be assessed through in-app feedback and documented changes in patient healthrelated data once recommendations were followed. Last, the usability of the user interface will be monitored based on app usage metrics derived from the user's behavior in the mobile application.

\subsection{Limitations}

Our study is limited in multiple ways despite the yet limited scope of a research in progress paper. First, healthcare-related IS underlie strong legal regulations, which are mostly country-specific. Therefore, the artifact must eventually be aware of its location to ensure legal compliance, e.g., regarding data protection and privacy regulations in the applicable law. Second, as the data collected from patients is intended to be used in drug research as a pointer towards relevant areas of study, the data collection needs to be safe against any type of manipulation. Hence, future research needs to target the security aspects of both the mobile application as well as the recommendation engine. Third, this architecture is limited to German users but is prospectively generalizable to other countries, thereby extending the user base to benefit from a larger number of users for more stable results for the recommender system. Fourth, this study is limited to CVD patients. However, we posit that architecture can be transferred to any sickness affected by female-specific body conditions, such as depression and diabetes. Fifth, there is still a lack of ready-to-use solutions for explainable AI. Accordingly, research is needed to develop individual explainable solutions for each specific recommendation. 


\section{References}

[1] L. Karlsson Lind et al., "Sex differences in drugs: the development of a comprehensive knowledge base to improve gender awareness prescribing", Biology of sex differences, 8 (1), 2017.

[2] I. Spoletini et al., "Sex differences in drug effects: interaction with sex hormones in adult life", Handbook of experimental pharmacology (214), 2012, pp. 91-105.

[3] V. Regitz-Zagrosek, "Geschlechterunterschiede in der Pharmakotherapie", Bundesgesundheitsblatt, Gesundheitsforschung, Gesundheitsschutz, 57 (9), 2014, pp. 1067-1073.

[4] D. Gemmati et al., "''Bridging the Gap" Everything that Could Have Been Avoided If We Had Applied Gender Medicine, Pharmacogenetics and Personalized Medicine in the Gender-Omics and Sex-Omics Era", International journal of molecular sciences, 21 (1), 2019.

[5] V. Raparelli et al., "Treatment and Response to Statins: Gender-related Differences", Current medicinal chemistry, 24 (24), 2017, pp. 2628-2638.

[6] S.-E. Kim et al., "Sex- and gender-specific disparities in colorectal cancer risk", World journal of gastroenterology, 21 (17), 2015, pp. 5167-5175.

[7] A. Di Paolo et al., "Personalized medicine in Europe: not yet personal enough?", BMC health services research, 17 (1), 2017, p. 289.

[8] A. Pokorska-Bocci et al., '"Personalized medicine': what's in a name?", Personalized medicine, 11 (2), 2014, pp. 197-210.

[9] I. Ekman et al., "Person-centered care--ready for prime time", European journal of cardiovascular nursing, 10 (4), 2011, pp. 248-251.

[10] G. Elwyn et al., "Shared Decision Making: A Model for Clinical Practice", Journal of General Internal Medicine, 27 (10), 2012, pp. 1361-1367.

[11] L. Li and X.-j. Tang, "A Solution to the Cold-Start Problem in Recommender Systems Based on Social Choice Theory", In K. Lavangnananda et al. (eds.), Intelligent and Evolutionary Systems, Springer International Publishing, Cham, 2016, pp. 267-279.

[12] Z. Zhong and Y. Li, "A Recommender System for Healthcare Based on Human-Centric Modeling", In 2016 IEEE 13th International Conference on e-Business Engineering (ICEBE), IEEE, Macau, 2016, pp. 282-286.

[13] A. Gyrard and A. Sheth, "IAMHAPPY: Towards an IoT knowledge-based cross-domain well-being recommendation system for everyday happiness", Smart Health, 15, 2020

[14] R. Burke, "Hybrid Recommender Systems: Survey and Experiments", User Modeling and User-Adapted Interaction, 12 (4), 2002, pp. 331-370.

[15] J. Basiri et al., "Alleviating the cold-start problem of recommender systems using a new hybrid approach", In 2010 5th International Symposium on Telecommunications, IEEE, Tehran, 2010, pp. 962-967.

[16] Y. Pérez-Gallardo et al., "Collective intelligence as mechanism of medical diagnosis: The iPixel approach", Expert Systems with Applications, 40 (7), 2013, pp. $2726-2737$
[17] K. Kourou et al., "Machine learning applications in cancer prognosis and prediction", Computational and structural biotechnology journal, 13, 2015, pp. 8-17.

[18] C. M. Bishop, "Pattern recognition and machine learning": Springer. New York, 2013.

[19] R. Guidotti et al., "A Survey of Methods for Explaining Black Box Models", ACM Comput. Surv., 51 (5), 2019, pp. 1-42.

[20] H. K. Dam, T. Tran, and A. Ghose, "Explainable software analytics", In Proceedings of the 40th International Conference on Software Engineering New Ideas and Emerging Results, ACM Press, Gothenburg, 2018, pp. 53-56.

[21] C. Eiras-Franco et al., "A scalable decision-tree-based method to explain interactions in dyadic data", Decision Support Systems, 127, 2019.

[22] Y. Yang et al., "Explaining Therapy Predictions with Layer-Wise Relevance Propagation in Neural Networks", In 2018 IEEE International Conference on Healthcare Informatics (ICHI), IEEE, New York, 2018, pp. 152-162.

[23] K. Peffers et al., "A Design Science Research Methodology for Information Systems Research", Journal of Management Information Systems, 24 (3), 2007, pp. 45-77.

[24] B. Sleath et al., "Physician-patient communication about over-the-counter medications", Social Science \& Medicine, 53 (3), 2001, pp. 357-369.

[25] N. Goodwin, "Towards People-Centred Integrated Care: From Passive Recognition to Active Co-production?", International journal of integrated care, 16 (2), 2016, p. 15.

[26] J. Håkansson Eklund et al., "''Same same or different?" A review of reviews of person-centered and patientcentered care", Patient education and counseling, 102 (1), 2019, pp. 3-11.

[27] Z. Khanzadeh and M. Mahdavi, "Solving Cold Start Problem in Collaborative Filtring Method of Recommender Systems", ICEB 2010 Proceedings, 66, 2010.

[28] J. Webster and R. T. Watson, "Analyzing the Past to Prepare for the Future: Writing a Literature Review", MIS Quarterly, 26 (2), 2002, pp. xiii-xxiii.

[29] S. Gregor and A. R. Hevner, "Positioning and Presenting Design Science Research for Maximum Impact", MISQ, 37 (2), 2013, pp. 337-355.

[30] A. R. Gagliardi et al., "How do and could clinical guidelines support patient-centred care for women: Content analysis of guidelines", PloS one, 14 (11), 2019.

[31] J. U. Ramlakhan et al., "What constitutes patient-centred care for women: a theoretical rapid review", International journal for equity in health, 18 (1), 2019.

[32] M. C. Meijers et al., "Shared decision-making in general practice: an observational study comparing 2007 with 2015", Family practice, 36 (3), 2019, pp. 357-364.

[33] D. M. Nestler et al., "Does gender bias in cardiac stress testing still exist? A videographic analysis nested in a randomized controlled trial", The American journal of emergency medicine, 35 (1), 2017, pp. 29-35.

[34] N. Huba and Y. Zhang, "Designing patient-centered personal health records (PHRs): health care professionals' 
perspective on patient-generated data", Journal of medical systems, 36 (6), 2012, pp. 3893-3905.

[35] C. L. Petersen et al., "Development and Implementation of a Person-Centered, Technology-Enhanced Care Model For Managing Chronic Conditions: Cohort Study", JMIR mHealth and uHealth, 7 (3), 2019.

[36] Reis, L., Maier, C., Mattke, J., \& Weitzel, T., "Chatbots in Healthcare: Status Quo, Application Scenarios for Physicians and patients and Future Directions", ECIS, 2020.

[37] K. Gai et al., "Ontology-Based Knowledge Representation for Secure Self-Diagnosis in PatientCentered Teleheath with Cloud Systems", In 2015 IEEE 2nd International Conference on Cyber Security and Cloud Computing, IEEE, New York, 2015, pp. 98-103.

[38] M. Radha et al., "Lifestyle Recommendations for Hypertension through Rasch-based Feasibility Modeling", In Proceedings of the 2016 Conference on User Modeling Adaptation and Personalization, ACM Press, Halifax, 2016, pp. 239-247.

[39] A. Ema, K. Nagakura, and T. Fujita, "Proposal for Type Classification for Building Trust in Medical Artificial Intelligence Systems", In Proceedings of the AAAI/ACM Conference on AI, Ethics, and Society, ACM, New York, 2020, pp. 251-257.

[40] L. Oliva-Felipe et al., "Health Recommender System design in the context of CAREGIVERSPRO-MMD Project", In Proceedings of the 11th PErvasive Technologies Related to Assistive Environments Conference, ACM, Corfu, 2018, pp. 462-469.

[41] N. Mesbah and L. Pumplun, "“Hello, I'm here to help you" - Medical care where it is needed most: Seniors' acceptance of health chatbots," Darmstadt Technical University, 2020.

[42] Y.-K. Lin et al., "Healthcare Predictive Analytics for Risk Profiling in Chronic Care: A Bayesian Multitask Learning Approach", MISQ, 41 (2), 2017, pp. 473-495.

[43] A. O. Afolabi and P. Toivanen, "Integration of Recommendation Systems Into Connected Health for Effective Management of Chronic Diseases", IEEE Access, 7, 2019, pp. 49201-49211.

[44] P. D. Kaur and I. Chana, "Cloud based intelligent system for delivering health care as a service", Computer methods and programs in biomedicine, 113 (1), 2014, pp. 346-359.

[45] E. Carrodeguas et al., "Use of Machine Learning to Identify Follow-Up Recommendations in Radiology Reports", Journal of the American College of Radiology : JACR, 16 (3), 2019, pp. 336-343.

[46] E. Tjoa and C. Guan, "A Survey on Explainable Artificial Intelligence (XAI): Towards Medical XAI,” 2019.

[47] H. Avdiji and R. Winter, "Knowledge Gaps in Design Science Research", In International Conference on Information Systems (ICIS), Munich, 2019.

[48] Johannes Schobel et al., "Development of Mobile Data Collection Applications by Domain Experts: Experimental Results from a Usability Study", In E. Dubois and K. Pohl (eds.), Advanced Information Systems Engineering, Springer, Cham, 2017.

[49] C. C. Lamb, Y. Wang, and K. Lyytinen, "Shared decision making: Does a physician's decision-making style affect patient participation in treatment choices for primary immunodeficiency?", Journal of evaluation in clinical practice, 25 (6), 2019, pp. 1102-1110.

[50] Y. Ou et al., "Automatic Drug Pills Detection based on Convolution Neural Network", In 2018 International Conference on Orange Technologies (ICOT), Bali, 2018, pp. $1-4$.

[51] S. Russo et al., "Understanding Patients' Preferences: A Systematic Review of Psychological Instruments Used in Patients' Preference and Decision Studies", Value in health: the journal of the International Society for Pharmacoeconomics and Outcomes Research, 22 (4), 2019, pp. 491-501.

[52] J.Archenaa and E.A.Mary Anita, "Health Recommender System using Big data analytics", Journal of Management Science and Business Intelligence, 2 (2), 2017, pp. 17-24.

[53] A. Kaplun et al., "PGMD: a comprehensive manually curated pharmacogenomic database", The Pharmacogenomics Journal, 16 (2), 2016, pp. 124-128.

[54] Medscape, Drug Interaction Checker. https://reference.medscape.com/drug-interactionchecker (Accessed: 13-07-20).

[55] G. A. Tsihrintzis, M. Virvou, and L. C. Jain, "Multimedia Services in Intelligent Environments: Advances in Recommender Systems", In G. A. Tsihrintzis et al. (eds.), Multimedia Services in Intelligent Environments. 2013 edn., Springer, Cham, 2013, pp. 1-5.

[56] F. North and R. Chaudhry, "Apple HealthKit and Health App: Patient Uptake and Barriers in Primary Care", Telemedicine journal and e-health : the official journal of the American Telemedicine Association, 22 (7), 2016, pp. 608-613.

[57] M. Gil et al., "Towards a Knowledge-Based Recommender System for Linking Electronic Patient Records With Continuing Medical Education Information at the Point of Care", IEEE Access, 7, 2019, pp. 1595515966.

[58] X. Deng and F. Huangfu, "Collaborative Variational Deep Learning for Healthcare Recommendation", IEEE Access, 7, 2019, pp. 55679-55688.

[59] M. Huang et al., "A Clinical Decision Support Framework for Heterogeneous Data Sources", IEEE journal of biomedical and health informatics, 22 (6), 2018, pp. 1824-1833.

[60] L. R. Ferretto et al., "A Physical Activity Recommender System for Patients With Arterial Hypertension", IEEE Access, 8, 2020, pp. 61656-61664.

[61] R. W. Gregory et al., "The Role of Artificial Intelligence and Data Network Effects for Creating User Value", AMR, 2020.

[62] Z. Che et al., "Interpretable Deep Models for ICU Outcome Prediction", AMIA Annual Symposium Proceedings, 2017, pp. 371-380.

[63] M. A. Kohl et al., "Explainability as a Non-Functional Requirement", In 2019 IEEE 27th International Requirements Engineering Conference, IEEE, Jeju Island, 2019, pp. 363-368.

[64] Hevner et al., "Design Science in Information Systems Research", MIS Quarterly, 28 (1), 2004. 